\title{
The influence of feedback from massive stars on the formation and emergence of massive clusters
}

\author{
James E. Dale $\mathbf{e}^{1,2}$ \\ ${ }^{1}$ Universitäts-Sternwarte München, Scheinerstr. 1, 81679 München, Germany. \\ ${ }^{2}$ Excellence Cluster 'Universe', Boltzmannstr. 2, 85748 Garching, Germany. \\ email: dale.james.e@gmail.com
}

\begin{abstract}
Massive star clusters are of fundamental importance both observationally, since they are visible at such great distances, and theoretically, because of their influence on the large-scale ISM. Understanding stellar feedback is a prerequisite for making sense of their formation and early evolution, since feedback influences cluster structure, star formation efficiency, and sets the timescales on which clusters emerge from their parent clouds to become optically visible. I review the progress made in understanding these issues from a numerical perspective.
\end{abstract}

Keywords. stars: formation, galaxies: star clusters, ISM: kinematics and dynamics

\section{Introduction}

Throughout most of the history of the Universe, star formation has occurred in a hierarchically-clustered fashion. Dispersal of the more loosely bound majority of stellar structures result in field galactic stellar populations, while the most strongly-bound minority survive as long-lived stellar clusters [Kruijssen (2012a)].

Owing to their rapid evolution, massive stars are mostly to be found in stellar associations, both those destined to be dispersed and those that will remain bound. This has important implications for the evolution of the interstellar medium (ISM), the structure of galaxies and the reionisation of, and distribution of metals within, the early Universe, since the effects of feedback from an evenly-distributed population of sources are very different from those of a clustered population.

As well as their central role in ISM dynamics, clusters are an invaluable observational tool, providing a window into the evolution of the ISM over a large fraction of the age of the Universe [e.g. Adamo et al. (2011)]. However, the interpretation of observations of unresolved clusters relies on a detailed understanding of how their spectral energy distributions (SEDs) evolve during the most complex phase of their lives. There are many physical processes contributing to the evolution of both the intrinsic and apparent brightnesses and SEDs of clusters, including the triggering, termination and overall rate and cadence of star formation, the clearing of gas from the cluster volumes, and the disruption of the parent giant molecular clouds (GMCs). Worryingly (in view of the importance of clusters) these processes are very poorly understood.

What is clear is that feedback is a central plank in a comprehensive model of star formation, and is sufficiently complex that numerical simulations are an indispensable tool in helping to understand it. In particular, the task of explaining how the many different mechanisms interact with one another is best achieved with the aid of computations. This is especially true of primordial, or Population III star formation where at present, 
we have almost no observational data to guide us.

\section{Population III vs. Population II star formation}

The very first generation of stars have as yet not been observed (they are one of the principal targets for the forthcoming James Webb Space Telescope), but theoretical considerations demonstrate that they formed in a very different fashion from their descendants. While most star formation has occurred through gravitational fragmentation in GMCs within galaxies occupying dark matter haloes, the first stars formed before the existence of either molecular gas or galaxies. Pop III stars formed from baryons accumulating in $\sim 10^{6} \mathrm{M}_{\odot}$ dark-matter minihaloes with virial radii of $\sim 100 \mathrm{pc}$. Without any molecules except $\mathrm{H}_{2}$, these systems are less able to cool than GMCs, and have higher temperatures and Jeans masses. As a result, Pop III stars were expected to be considerably more massive than present-day objects [e.g. Abel et al. (2002), Hosokawa \& Omukai (2009)] and, owing to the difficulty of fragmentation, were thought to form alone and not in clusters.

However, recent 3-D simulations with more advanced treatments of thermodynamics find that angular momentum conservation and fragmentation result in the formation of Pop III clusters [e.g. Clark et al. (2008), Stacy et al. (2010), Greif et al. (2011), Smith et al. (2011)], both increasing the numbers of low-mass stars and limiting the growth of the central objects (fragmentation-induced starvation - Peters et al. 2010, Smith et al. 2011). This steepening of the Pop III IMF dramatically reduces the quantity of feedback produced per unit stellar mass.

\section{Feedback mechanisms through cosmic time}

One of the chief difficulties in modelling and understanding feedback is that there are many different mechanisms operating on widely different length- and timescales, which interact with other in a complex and non-linear manner. There has been considerable progress in recent years in understanding the individual mechanisms, as described below. However, no simulation including all of them has yet been attempted.

\subsection{Accretion feedback}

All stars exert feedback as they form via thermal accretion feedback and jets. Thermal feedback derives from the conversion of gravitational potential energy to heat as accreting material falls onto the protostar, and from the Kelvin-Helmholtz contraction of the protostar itself (some models, e.g. [Offner et al. (2009a)], also include the energy released by deuterium burning). The infrared radiation released is absorbed by dust and, where the gas-dust mixture is dense enough, couples to the gas. Accretion heating in primordial star formation has been modelled (e.g. [Clark et al. (2008), Clark et al. (2011), Smith et al. $(2011,2012)$ and has only a minimal effect, heating the gas only modestly and leaving fragmentation largely unchanged. This is partly due to higher background temperatures of primordial gas, but the absence of dust can also disable accretion heating.

On the contrary, accretion heating in present-day star formation results in lower accretion rates and suppression of fragmentation (e.g. [Bate (2009, 2012, 2014), Offner et al. (2009a), Urban et al. (2010), Krumholz et al. (2010, 2011, 2012)].

Jets are also driven by accretion, and their short cooling times allow them to be thought 
of as depositing chiefly momentum. As far as the author is aware, jets from primordial stars have not been modelled, but their main effects on present-day star formation are to limit accretion onto individual objects and to drive small-scale turbulence (e.g. [Carroll et al. (2009), Offner \& Arce (2014), Federrath et al. (2014)]. Because they are both driven by, and have influence on, the accretion process, the interaction of accretion heating and jets can be complex (e.g. [Krumholz et al. (2012), Hansen et al. (2012)], lessening each others' effectiveness. This highlights the issue that different feedback mechanisms are not independent and their effects are not necessarily additive.

It might be argued that the range of influence of accretion feedback is too small to be of any consequence for an entire cluster, but this is not the case. [Krumholz et al. $(2010,2011,2012)]$ show that it is a necessary but not sufficient condition for obtaining the correct IMF robustly. [Bate (2009, 2012), Stamatellos et al. (2012)] demonstrate the importance of radiative feedback in particular on the low-mass end of the IMF. Accretion feedback also affects the environments massive stars are born into, in the sense of helping set the star formation efficiency the clouds have achieved when the massive stars ignite.

\subsection{HII regions}

HII regions are the most studied form of stellar feedback. Owing to very low primordial metallicities, Pop III stars had higher surface temperatures, and cooling within their HII regions was less efficient, leading to higher HII region temperatures $(\approx 30 \mathrm{kK}$ as opposed to present-day values of $\approx 10 \mathrm{kK}$ ). This higher temperature is sufficient to ionise He and an important observational indicator of Pop III stars is expected to be simultaneous Ly $-\alpha$ and He II emission (e.g. [Johnson, (2009)]. Primordial HII region temperatures were sufficient to expel baryons from minihaloes, allowing large fractions of the ionising photons to escape and play their part in cosmic reionisation (e.g. [Yoshida et al. (2007)]. Anisotropic accretion was nevertheless able to continue, as in modern star formation (e.g. [Tan \& McKee (2008), Hosokawa et al. (2012), Stacey et al. (2012)], but the effectiveness of feedback depends strongly on the uncertain shape of the Pop III IMF. Radiative feedback from massive Pop III stars suppresses star formation very effectively, but that from normal-mass ones does not (e.g. [Jeon et al. (2015)]).

Present-day simulations of feedback have largely concentrated on HIIRs and agreement is emerging that $\sim 10^{4} \mathrm{M}_{\odot}$ clouds can be severely damaged or destroyed by HII regions on Myr timescales ([Arthur et al. (2011), Walch et al. (2012), Dale et al. (2012), Colín et al.(2013)]. However, as suggested by, e.g. [Matzner (2002)] and confirmed by [Dale et al. $(2012,2013,2014)]$, massive clouds with higher escape velocities are more resilient. This is shown qualitatively in Figure 1, which depicts the effects of $\approx 3 \mathrm{Myr}$ of photoionisation and wind feedback on a series of molecular clouds ranging in mass from $10^{4}-10^{6} \mathrm{M}_{\odot}$.

\subsection{Stellar winds}

Stellar winds from massive main-sequence stars have received comparatively little attention as feedback agents. Since they are driven by metal lines in stellar atmospheres, winds were absent in Pop III stars, and are generally weaker in stars with low metallicity. The dynamically-relevant quantities are the rates of release of momentum $\dot{p}=\dot{M} v_{\infty}$ and mechanical energy $L=0.5 \dot{M} v_{\infty}^{2}$, which depend on the intrinsic stellar properties $\dot{M}$ (the mass loss rate) and $v_{\infty}$ (the wind terminal velocity). Both of these in turn depend on the metallicity. At moderately low metallicity $(\gtrsim 0.01)$ [Vink et al. (2001)] deduce $\dot{M} \propto Z^{0.7-0.8}$ and [Leitherer et al. (1992)] find $v_{\infty} \propto Z^{0.13}$. [Kudritski (2002)] find that steeper relations apply at lower metallicities. 


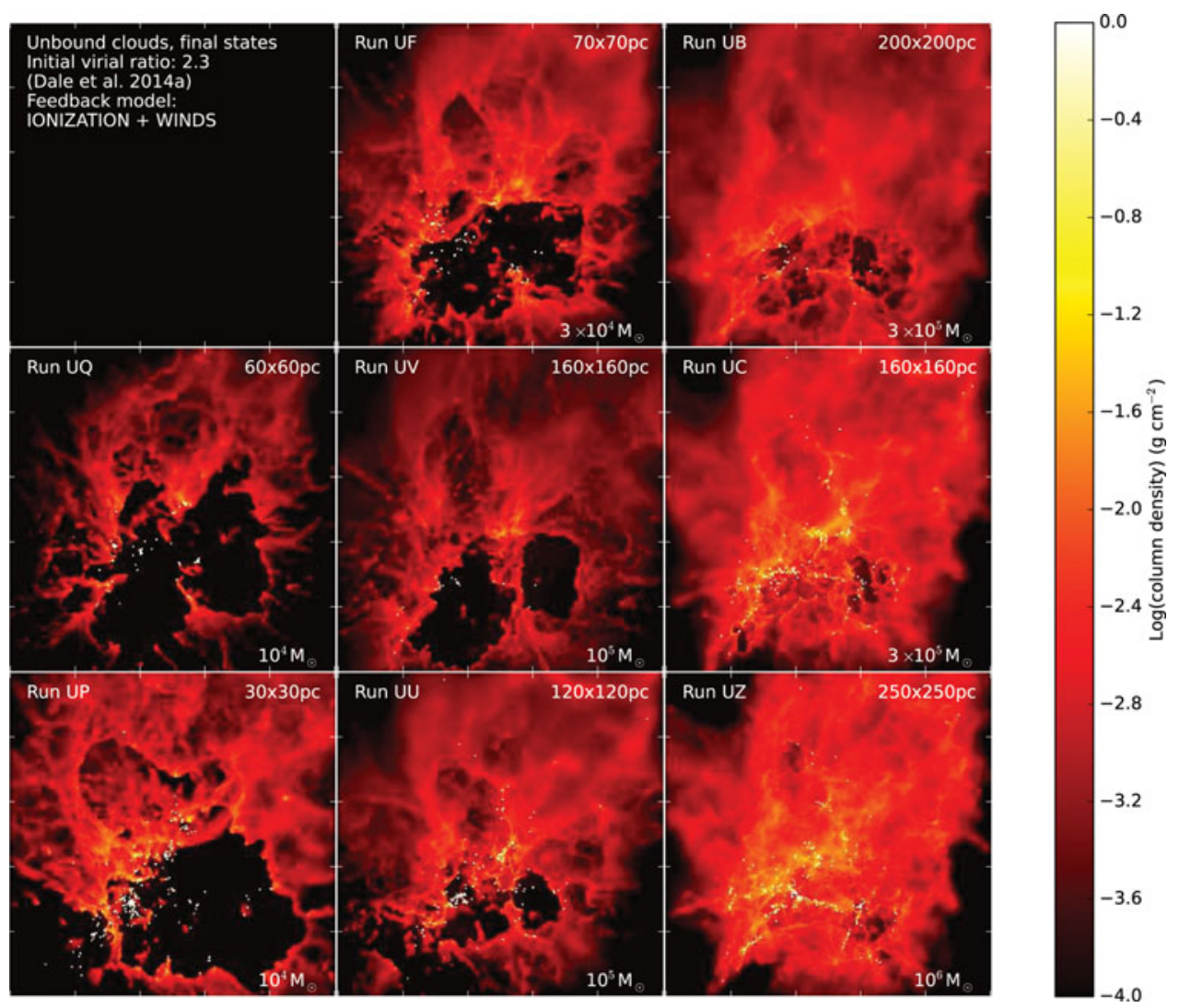

Figure 1. Column-density images of $10^{4}-10^{6} \mathrm{M}_{\odot}$ turbulent clouds after $\approx 3 \mathrm{Myr}$ of photoionisation and wind from Dale et al. (2014). Image sizes are given in the top right corner, and clouds masses in the bottom right.

The influence of winds on modern star formation is much debated. [Rogers \& Pittard (2013)] find that winds open channels through the cloud and leak, so that merely injecting energy is not sufficient to disrupt a cloud - the cloud must retain the energy long enough for a large fraction of it to be converted to bulk kinetic energy. [Rosen et al. (2014)] evaluated, for four young systems (30 Dor, M17, Carina, NGC 3603), how much wind energy had been injected, and what fraction had been converted to work, finding that this was generally rather low, $\sim 10 \%$, and inferring that much of the energy was lost due to leakage of hot gas. This is in line with a large body of earlier research (e.g. [Garcia-Segura \& Mac Low (1995), Oey (1996), Naze et al. (2002), Dopita et al. (2005)] suggesting that wind bubbles expand slower than spherical models predict.

\subsection{Supernovae}

On the largest scales, supernovae ( $\mathrm{SNe}$ ) are the most important stellar feedback mechanism. Pop III SNe were thought to be unusually violent, since $140-260 \mathrm{M}_{\odot}$ stars die as pair-instability SNe, releasing $10^{53} \mathrm{erg}$ (e.g. [Heger \& Woosley (2002), Whalen et al. (2008a), Greif (2015)]. Whether and how often these occur depends on the Pop III IMF, which is still very uncertain. What is clear is that the pre-SN action of photoionisation governs the effectiveness of supernovae - without it, SNe explode in dense material which rapidly quenches them. However, as described above, photoionisation on its own is able 
to expel baryons from primordial minihaloes. This allows the SNe to enter their radiative phases much later and to expand to much larger distances and distribute metals over much larger volumes (e.g. [Whalen et al. (2008b), Jeon et al. (2014)].

A similar phenomenon is observed in present-day models, which demonstrate that the environments in which SNe explode completely control their influence. [Iffrig \& Hennebelle (2014)] model SN detonations inside and outside clouds and find very different results in terms of how much dense gas survives the explosion. [Walch \& Naab (2015)] examined the effect of photoionisation acting before the SN, finding that pre-ionisation results in $\sim 50 \%$ more momentum being deposited.

\section{Summary}

Accretion-driven feedback is likely less effective at early epochs than in present-day star formation, where it plays an important role in moderating star formation rates and fragmentation and is one of the main processes regulating the IMF. Similarly, main sequence winds were absent in primordial star formation owing to the lack of driving metal ions in stellar atmospheres. Their influence on later star formation is still unclear, but both observations and simulations suggest that their effectiveness is strongly reduced by leakage of hot gas from clouds.

Photoionisation has been of central importance at all redshifts, operating on very similar physical principles, modulo the higher HIIR temperatures at high redshift. However, its effectiveness depends strongly on halo/GMC escape velocities, and on the Pop III IMF at high redshifts. While most primordial minihaloes were vulnerable to photoionisation, many present-day GMCs are not and other mechanisms such as radiation pressure need to be invoked to explain the very young, dense gas-free clusters observed by, e.g. [Hollyhead et al.(2015)]. This issue is now receiving attention [Skinner \& Ostriker (2015)].

Supernovae have likewise been important at all redshifts, albeit in different senses. Today, SNe help regulate the cycling of matter within galaxies, but bottom-up hierarchical structure formation implies that Pop III (and possibly Pop II) star formation was well underway before galactic assembly, so that feedback from Pop III (and possibly early Pop II) stars influenced the formation of galaxies (e.g. [Bromm \& Yoshida, (2011)]. The details of the role of SNe depend, again, on the Pop III IMF, which sets the proportion of PISNe. At all times, the effectiveness of SNe depends very strongly on the earlier-acting feedback processes, especially photoionisation. While Pop III SNe likely played little part in expelling baryons from minihaloes (since ionisation was able to accomplish this alone), present-day SNe may assist in disrupting molecular clouds, although observations of gasfree clusters too young to have hosted SNe imply that this is certainly not always the case.

Simulations suggest that feedback-triggered star formation has been operating throughout much of the Universe's history, although it seems to be of only second-order importance today, and the identification of triggered stars may be very difficult (e.g. [Dale et al. (2015b)]. Several primordial calculations suggest that Pop III and Pop II star formation could be contemporaneous and that Pop II formation can be triggered by Pop III stars (e.g. [Pawlik et al., (2013), Jeon et al., (2015)]. Conversely, [Wise et al. (2012)] showed that patchy IGM pollution allows Pop III formation to continue in pockets up to $\mathrm{z}=6$. These effects may in principle be easier to deduce observationally.

The interplay between star formation, gas expulsion and cloud disruption is still not well understood. This emergence timescale is important, since clusters are brightest at around the time when they are clearing away residual gas, before the first SNe. Observations (e.g. [Hollyhead et al. (2015)] find this timescale to be short, 4Myr even for massive 
$\left(>10^{4} \mathrm{M}_{\odot}\right)$ clusters. [Dale et al. $(2015 \mathrm{a})$ ] are able to reproduce such short timescales only for substantially lower-mass $\left(\sim 10^{3} \mathrm{M}_{\odot}\right)$ clusters.

While important observationally, gas expulsion form clusters appears to have only modest dynamical influence. Simulations by [Offner et al. (2009b), Girichidis et al. (2011), Kruijssen et al., (2012b)] find that stars rapidly decouple from the gas, forming stellardominated subsystems largely immune to gas expulsion/cloud dispersal. [Dale et al. (2015a)] observed that, even where feedback was able to expel large fractions of the gas from cloud potential wells, the fractions of stars unbound were much more modest.

In general, many of the processes that influence the evolution of clusters have done so in a very similar fashion throughout the history of the Universe, and primordial and present-day star formation are not as different as they may at first appear. These different processes interact with one another in a complex and, as yet, only dimly understood fashion, and this topic will continue to be a major focus of star formation research for the foreseeable future.

\section{References}

Abel, T., Bryan, G., \& Norman, M., 2002, Science, 295, 93

Adamo, A., Östlin, G., \& Zackrisson, E., 2011, MNRAS, 417, 1904

Arthur, S. J., Henney, W. J., Mellema, G., de Colle, F., \& Vázquez-Semadeni, E., 2011, MNRAS, 414,1747

Bate, M. R., 2009, MNRAS, 392, 1363

Bate, M. R., 2012, MNRAS, 419, 3115

Bate, M. R., 2014, MNRAS, 442, 285

Bromm, V. \& Yoshida, N., 2011, ARA $\& A, 49,373$

Carroll, J. J., Frank. A., Blackman, E. G., Cunningham, A. J., \& Quillen, A. C., 2009, ApJ, 695,1376

Clark, P. C., Glover, S. C. O., \& Klessen, R. S., 2008, ApJ, 672, 757

Clark, P. C., Glover, S. C. O., Smith, R. J., Greif, T. H., Klessen, R. S., \& Bromm, V., 2011, Science, 331, 1040

Colín, P., V azquez-Semadeni, E., \& Gomez, G. C., 2013, MNRAS, 435, 1701

Dale, J. E., Ercolano, B., \& Bonnell, I. A., 2012, MNRAS, 424, 377

Dale, J. E., Ercolano, B., \& Bonnell, I. A., 2013, MNRAS, 430, 234

Dale, J. E., Ngoumou, J., Ercolano, B., \& Bonnell, I. A., 2014, MNRAS, 442, 694

Dale, J. E., Ercolano, B., \& Bonnell, I. A., 2015, MNRAS, 451, 987

Dale, J. E., Haworth, T. H., \& Bressert, E., 2015, MNRAS, 450, 1199

Dopita, M. A., Groves, B. A., Fischera, J., Sutherland, R. S., Tuffs, R J., Popescu, C. C., Kewley, L. J., Reuland, M., \& Leitherer, C., 2005, ApJ, 619, 755

Federrath, C., Schrön, M., Banerjee, R., \& Klessen, R. S., 2014, ApJ, 790, 128

Garcia-Segura, G. \& Mac Low, M-M., 1995, ApJ, 445, 145

Girichidis, P., Federrath, C., Banerjee, R., \& Klessen, R. S., 2011, MNRAS, 413, 2741

Greif, T. H., Springel, V., White, S. D. M., Glover, S. C. O., Clark, P. C., Smith, R. J., Klessen, R. S., \& Bromm, V., 2011, ApJ, 737, 75

Greif, T. H., 2015, Computational Astrophysics 83 Cosmology, 2, 3

Hansen, C. F., Klein, R. I., McKee, C. F., \& Fisher, R. T., 2012, ApJ, 747, 22

Heger, A. \& Woosley, A. E., 2002, ApJ, 567, 532

Hollyhead, K., Bastian, N., Adamo, A., Silva-Villa, E., Dale, J., Ryon, J. E., \& Gazak, Z., 2015, MNRAS, 449, 1106

Hosokawa, T. \& Omukai, K., 2009, ApJ, 691, 823

Hosokawa, T., Yoshida, N., Omukai, K., \& Yorke, H. W., 2012, ApJ, 760, L37

Iffrig, O. \& Hennebelle, P., 2014, A\&A, 596, 95

Jeon, M., Pawlik, A. H., Bromm, V., \& Milosavljević, M., 2014, MNRAS, 444, 3288 
Jeon, M., Bromm, V., Pawlik, A. H., \& Milosavljević, M., 2015, MNRAS, 452, 1152

Johnson, J. L., 2010, MNRAS, 404, 1425

Krumholz, M. R., Cunningham, A. J., Klein, R. I., \& McKee, C. F., 2010, ApJ, 713, 1120

Krumholz, M. R., Klein, R. I., \& McKee, C. F., 2011, ApJ, 740, 74

Krumholz, M. R., Klein, R. I., \& McKee, C. F., 2012, ApJ, 754, 71

Kruijssen, J. M. D., 2012, MNRAS, 426, 3008

Kruijssen, J. M. D., Maschberger, T., Moeckel, N., Clarke, C. J., Bastian, N., \& Bonnell, I. A., 2012, MNRAS, 419, 841

Kudritski, R. P., 2002, ApJ, 577, 389

Leitherer, C., Robert, C., \& Drissen, L., 1992, ApJ, 401, 596

Matzner, C. D., 2002, ApJ, 566, 302

Nazé, Y., Chu, Y. H., Guerrero, M. A., Oey, M. S., Gruendl, R. S., \& Smith, R. C., 2002, ApJ, 124,3325

Oey, M. S., 1996, ApJ, 467, 666

Offner, S. S. R., Klein, R. I., McKee, C. F., \& Krumholz, M. R., 2009, ApJ, 703, 131

Offner, S. S. R., Hansen, C. E., \& Krumholz, M. R., 2009, ApJ, 704, L124

Offner, S. S. R. \& Arce, H. G., 2014, ApJ, 784, 61

Pawlik, A, H., Milosavljević, M., \& Bromm, V., 2013, ApJ, 767, 59

Peters, T., Klessen, R. S., Mac Low, M-M., \& Banerjee, R., 2010, ApJ, 725, 134

Rogers, H. \& Pittard, J. M., 2013, MNRAS, 431, 1337

Rosen, A. L., Lopez, L. A., Krumholz, M. R., \& Ramirez-Ruiz, E., 2014, MNRAS, 442, 2701

Skinner, M. A. \& Ostriker, E. C., 2015, ApJ, 809, 187

Smith, R. J., Glover, S. C. O., Clark, P. C., Greif, T. H., \& Klessen, R. S., 2011, MNRAS, 414, 3633

Smith, R. J., Hosokawa, T., Omukai, K., Glover, S. C. O., \& Klessen, R. S., 2012, MNRAS, 424,457

Stacy, A., Greif, T. H., \& Bromm, V., 2010, MNRAS, 403, 45

Stacy, A., Greif, T. H., \& Bromm, V., 2012, MNRAS, 422, 290

Stamatellos, D., Whitworth, A. P., \& Hubber, D. A., 2012, MNRAS, 427, 1182

Tan, J. C. \& McKee, C. F., 2008, AIP Conf. Proc., 990, 47

Urban, A., Martel, H., \& Evans, N. J., 2010, ApJ, 710, 1343

Vink, J. S., de Koter, A., \& Lamers, H. J. G. L. M., 2001, A\&A, 369, 574

Walch, S. K., Whitworth, A. P., Bisbas, T. G., Wünsch, R., \& Hubber, D. A., 2012, MNRAS, 427,625

Walch, S. K. \& Naab, T., 2015, MNRAS, 451, 2757

Whalen, D., van Veelen, B., O'Shea, B. W., \& Norman, M. L., 2008, ApJ, 682, 49

Whalen, D., O'Shea, B. W., Smidt, J., \& Norman, M. L., 2008, ApJ, 679, 925

Wise, J. H., Turk, M. J., Norman, M. L., \& Abel, T., 2012, ApJ, 745, 50

Yoshida, N., Oh, S. P., Kitayama, T., \& Hernquist, L., 2007, ApJ, 663, 687 